\title{
Current Issues in Billing and Coding in Interventional Pain Medicine
}

\author{
Laxmaiah Manchikanti, MD
}

Interventional pain management is a dynamic field with changes occurring on a daily basis, not only with technology but also with regulations that have a substantial financial impact on practices. Regulations are imposed not only by the federal government and other regulatory agencies, and also by a multitude of other payors, state governments and medical boards. Documentation of medical necessity with coding that correlates with multiple components of the patient's medical record, operative report, and billing statement is extremely important.

Numerous changes which have occurred in the practice of interventional pain management in the new millennium continue to impact the financial viability of interventional pain practices along with patient access to these services. Thus, while complying with regulations of billing, coding and proper, effective, and ethical practice of pain management, it is also essential for physicians to understand financial aspects and the impact of various practice patterns.
This article provides guidelines which are meant to provide practical considerations for billing and coding of interventional techniques in the management of chronic pain based on the current state of the art and science of interventional pain management. Hence, these guidelines do not constitute inflexible treatment, coding, billing or documentation recommendations. It is expected that a provider will establish a plan of care on a case-bycase basis taking into account an individual patient's medical condition, personal needs, and preferences, along with physician's experience and in a similar manner, billing and coding practices will be developed. Based on an individual patient's needs, treatment, billing and coding, different from what is outlined here is not only warranted but essential.

Keywords: Interventional techniques, neural blockade, interventional pain management, medical necessity, billing, coding
While multiple issues of documentation, billing, and coding are facts of life for physicians practicing interventional pain management, emphasis continues on the description and definition of what the physician does for and to the patient. Various aspects of appropriate documentation, billing, and coding in interventional pain practice have been described earlier (1). Focus on errors for Medicare program as well as errors in other insurance programs, exclusion and sanction of medical providers, Medicare fraud hotline hits, qui tam cases and recoveries, and sky high settlements from institutions and individual physicians continue to increase. Recent developments include changes in CPT 2000 (2, 3); final rule for 2000, and proposed rule for 2001 on physician payment policies $(4,5)$; Medicare program prospective payment system for hospital outpatient services (6); program memorandum on the manner in

From Pain Management Center of Paducah, Paducah, Kentucky. Dr. Manchikanti is the medical director of Pain Management Center of Paducah. Address correspondence: Laxmaiah Manchikanti, MD, 2831 Lone Oak Road, Paducah, Kentucky 42003. Email: drm@ apex.net which the Health Care Financing Administration (HCFA) proposed to deal with this those deleted CPT codes for which replacement codes were created in CPT 2000 (7, 8); and HCFA's new position and acknowledgment that it would allow bilateral billing of new facet joint injection and transforaminal codes (2). In addition, the impact of national correct coding policy on interventional pain management (9-13), documentation of medical necessity, and Department of Justice - Health and Human Services (DOJHHS) health care fraud and abuse control program, continue to take the center stage.

Medicare program prospective payment system for hospital outpatient services; final rule: Department of Health and Human Services, Health Care Financing Administrations (6) has reduced reimbursement for interventional pain procedures for hospitals in a substantial manner which may have serious impact on patient access to interventional pain procedures (14-16). This is shown in Table 1.

HCFA, in its Ambulatory Surgery center proposal, eliminated all interventional pain medicine procedures, except for epidural and subarachnoid injections (17). However, 
Table 1. Comparison Payments Under the 2000 and 2001 Medicare Physician Fee Schedules and Hospital Outpatient Payment for 2000

\begin{tabular}{|c|c|c|c|c|c|c|}
\hline \multirow[b]{2}{*}{$\begin{array}{l}\text { CPT } \\
\text { Code }\end{array}$} & \multirow[b]{2}{*}{ Abbreviated Description of Procedure } & \multicolumn{2}{|c|}{2000 Physician Fee Schedule } & \multicolumn{3}{|c|}{2001 Proposed Rule } \\
\hline & & $\begin{array}{c}\text { Final Rule } \\
\text { CY 2000[i] } \\
\text { Nonfacility } \\
\text { Total } \\
\text { (\$) }\end{array}$ & $\begin{array}{c}\text { Final Rule } \\
\text { CY 2000[ii] } \\
\text { Facility } \\
\text { Total } \\
\text { (\$) }\end{array}$ & $\begin{array}{c}\text { Proposed Rule } \\
\text { CY 2001[iii] } \\
\text { Nonfacility } \\
\text { Total } \\
\text { (\$) }\end{array}$ & $\begin{array}{l}\text { Proposed Rule } \\
\text { CY 2001[iv] } \\
\text { Facility } \\
\text { Total } \\
\text { (\$) }\end{array}$ & $\begin{array}{c}\text { Proposed } \\
\text { Hospital } \\
\text { Outpatient } \\
\text { Payment } \\
\text { (\$) }\end{array}$ \\
\hline 20550 & Trigger-point injection & 72.86 & 41.36 & 86.77 & 43.57 & 102.31 \\
\hline 20600 & Small-joint injection & 56.75 & 35.51 & 64.44 & 38.08 & 102.31 \\
\hline 20605 & Intermediate-joint injection & 62.60 & 36.24 & 73.23 & 38.81 & 102.31 \\
\hline 20610 & Large-joint injection & 73.22 & 41.37 & 87.87 & 49.79 & 102.31 \\
\hline 27093 & Hip arthrography - without anesthesia & 250.44 & 75.06 & 354.79 & 72.50 & NA-N \\
\hline 27095 & Hip arthrography - with anesthesia & 261.78 & 85.67 & 370.53 & 82.38 & NA-N \\
\hline 27096 & SI-joint injection & 408.24 & 56.75 & 434.24 & 58.95 & NA-N \\
\hline 62263 & Percutaneous epidural adhesiolysis & 421.42 & 332.45 & 447.79 & 335.75 & 176.49 \\
\hline 62270 & Spinal puncture & 124.12 & 67.36 & 138.76 & 65.90 & 145.46 \\
\hline 62272 & Spinal puncture-for drainage of spinal fluid & 136.20 & 86.41 & 147.92 & 83.11 & 176.49 \\
\hline 62273 & Epidural blood patch & 128.51 & 123.75 & 129.98 & 124.12 & 176.49 \\
\hline 62280 & Neurolytic subarachnoid & 197.35 & 128.88 & 229.20 & 129.25 & 176.49 \\
\hline 62281 & Cervical/thoracic epidural-neurolytic & 184.53 & 130.71 & 207.60 & 128.51 & 176.49 \\
\hline 62282 & Lumbar/sacral epidural-neurolytic & 221.51 & 134.37 & 253.73 & 123.75 & 176.49 \\
\hline 62284 & Myelography & 163.29 & 101.78 & 190.03 & 98.49 & 176.49 \\
\hline 62287 & Decompression of nucleus pulposus & NA & 546.27 & NA & 529.43 & 676.88 \\
\hline 62290 & Lumbar diskography & 232.49 & 173.18 & 141.70 & 62.98 & NA-N \\
\hline 62291 & Cervica/thoracic diskography & 229.93 & 163.38 & 274.97 & 162.93 & NA-N \\
\hline 62292 & $\begin{array}{l}\text { Chemonucleolysis-single or multiple levels- } \\
\text { lumbar }\end{array}$ & NA & 556.16 & NA & 508.93 & 176.49 \\
\hline 62310 & Cervical/thoracic epidural & 198.08 & 93.36 & 197.35 & 94.83 & 176.49 \\
\hline 62311 & Lumbar/caudal epidural & 199.54 & 77.62 & 201.38 & 78.72 & 176.49 \\
\hline 62318 & Continuous epidural-cervical/thoracic & 206.86 & 101.05 & 206.50 & 102.88 & 176.49 \\
\hline 62319 & Continuous epidural-lumbar/sacral & 201.37 & 91.53 & 198.08 & 93.00 & 176.49 \\
\hline 62350 & Implantation of catheter & NA & 406.41 & NA & 403.48 & 307.41 \\
\hline 62355 & Removal of catheter & NA & 334.64 & NA & 323.67 & 307.45 \\
\hline 62360 & $\begin{array}{l}\text { Implantation or replacement of drug infusion } \\
\text { reservoir }\end{array}$ & NA & 162.56 & NA & 168.42 & 1235.45 \\
\hline 62361 & Implant of non-programmable pump & NA & 324.76 & NA & 325.50 & 1235.45 \\
\hline 62362 & Implant of programmable pump & NA & 429.84 & NA & 429.48 & 1235.45 \\
\hline 62365 & Removal of reservoir & NA & 347.46 & NA & 340.50 & 772.88 \\
\hline 63650 & Implantation of neurostimulator & NA & 462.80 & NA & 440.10 & 772.88 \\
\hline 63660 & Removal of neurostimulator & NA & 452.55 & NA & 416.30 & 772.88 \\
\hline 63685 & Implantation of pulse generator & NA & 511.49 & NA & 472.32 & 772.88 \\
\hline 63688 & Removal of pulse generator & NA & 401.65 & NA & 371.63 & 772.88 \\
\hline 64400 & Trigeminal nerve block & 91.90 & 53.08 & 106.55 & 56.02 & 160.98 \\
\hline 64402 & Facial nerve block & 132.54 & 69.57 & 155.24 & 67.37 & 160.98 \\
\hline 64405 & Greater occipital nerve block & 106.17 & 64.44 & 121.19 & 67.37 & 160.98 \\
\hline
\end{tabular}

[i]. Information obtained from the November 2, 1999 Federal Register. [ii]. Information obtained from the November 2, 1999 Federal Register. [iii]. Information obtained from the July 17, 2000 Federal Register. [iv]. Information obtained from the July 17, 2000 Federal Register. 
Table 1. (cont.) Comparison Payments Under the 2000 and 2001 Medicare Physician Fee Schedules and Hospital Outpatient Payment for 2000

\begin{tabular}{|c|c|c|c|c|c|c|}
\hline \multirow[b]{2}{*}{$\begin{array}{l}\text { CPT } \\
\text { Code }\end{array}$} & \multirow[b]{2}{*}{ Abbreviated Description of Procedure } & \multicolumn{2}{|c|}{2000 Physician Fee Schedule } & \multicolumn{3}{|c|}{2001 Proposed Rule } \\
\hline & & $\begin{array}{c}\text { Final Rule CY } \\
\text { 2000[i] } \\
\text { Nonfacility } \\
\text { Total } \\
\text { (\$) }\end{array}$ & $\begin{array}{c}\text { Final Rule } \\
\text { CY 2000[ii] } \\
\text { Facility } \\
\text { Total } \\
\text { (\$) }\end{array}$ & $\begin{array}{c}\text { Proposed Rule } \\
\text { CY 2001[iii] } \\
\text { Nonfacility } \\
\text { Total } \\
\text { (\$) }\end{array}$ & $\begin{array}{c}\text { Proposed } \\
\text { Rule CY } \\
\text { 2001[iv] } \\
\text { Facility Total } \\
\text { (\$) }\end{array}$ & $\begin{array}{c}\text { Proposed } \\
\text { Hospital } \\
\text { Outpatient } \\
\text { Payment } \\
\text { (\$) }\end{array}$ \\
\hline 64418 & Suprascapular nerve block & 108.01 & 65.17 & 119.00 & 68.10 & 160.98 \\
\hline 64420 & Intercostal nerve block-single & 99.22 & 63.34 & 112.40 & 60.05 & 160.98 \\
\hline 64421 & Intercostal nerve block-multiple & 128.88 & 88.23 & 142.79 & 83.85 & 160.98 \\
\hline 64425 & Ilioinguinal nerve block & 119.72 & 86.77 & 133.27 & 85.31 & 160.98 \\
\hline 64445 & Injection, anesthetic agent; sciatic nerve & 121.92 & 68.47 & 144.62 & 72.13 & 160.98 \\
\hline 64450 & Peripheral nerve block & 88.60 & 60.77 & 97.39 & 64.07 & 160.98 \\
\hline 64470 & Facet injection-cervical/thoracic-single & 203.93 & 106.17 & 204.67 & 90.44 & 160.98 \\
\hline 64472 & Facet injection-cervical/thoracic-additional & 170.98 & 63.34 & 170.99 & 64.07 & 160.98 \\
\hline 64475 & Facet injection-lumbar/sacral-single & 181.23 & 68.10 & 181.60 & 69.20 & 160.98 \\
\hline 64476 & Facet injection-lumbar/sacral-additional & 171.35 & 47.23 & 169.52 & 47.96 & 160.98 \\
\hline 64479 & Transforaminal cervical/thoracic-single & 221.51 & 106.17 & 221.88 & 108.38 & 160.98 \\
\hline 64480 & Transforaminal cervical/thoracic-additional & 199.54 & 73.59 & 198.45 & 74.69 & 160.98 \\
\hline 64483 & Transforaminal lumbar/sacral-single & 204.30 & 90.43 & 204.67 & 92.27 & 160.98 \\
\hline 64484 & Transforaminal lumbar/sacral-additional & 188.56 & 63.70 & 187.46 & 64.81 & 160.98 \\
\hline 64505 & Sphenopalatine ganglion block & 103.61 & 65.53 & 115.70 & 68.83 & 160.98 \\
\hline 64510 & Stellate ganglion block & 102.51 & 65.90 & 113.87 & 61.88 & 160.98 \\
\hline 64520 & Lumbar or thoracic sympathetic block & 129.61 & 72.12 & 152.31 & 68.10 & 160.98 \\
\hline 64530 & Celiac plexus block & 134.73 & 91.16 & 145.72 & 83.48 & 160.98 \\
\hline 64600 & Neurolytic-trigeminal-small branches & 226.63 & 206.50 & 238.36 & 208.33 & 160.98 \\
\hline 64605 & Neurolytic-trigeminal-2/3 division & 316.34 & 296.20 & 332.08 & 303.53 & 160.98 \\
\hline 64610 & Neurolytic-trigeminal-at foramen ovale & NA & 528.33 & NA & 491.36 & 160.98 \\
\hline 64612 & Neurolytic block-muscles of facial nerve & 156.70 & 120.45 & 163.30 & 134.74 & 160.98 \\
\hline 64613 & Neurolytic block-cervical spinal muscles & 139.49 & 120.82 & 139.86 & 133.64 & 160.98 \\
\hline 64620 & Intercostal neurolysis & 183.43 & 141.69 & 197.71 & 138.77 & 160.98 \\
\hline 64622 & Facet neurolysis-lumbar/sacral -single & 227.73 & 166.22 & 137.67 & 46.87 & 160.98 \\
\hline 64623 & Facet neurolysis-lumbar/sacral -additional & 111.67 & 59.68 & 129.98 & 53.82 & 160.98 \\
\hline 64626 & Facet neurolysis-cervical/thoracic-single & 259.59 & 161.10 & 261.42 & 162.56 & 160.98 \\
\hline 64627 & Facet neurolysis-cervical/thoracic-additional & 154.87 & 57.11 & 155.24 & 57.48 & 160.98 \\
\hline 64630 & Pudenal nerve & 201.00 & 165.86 & 104.35 & 47.23 & 160.98 \\
\hline 64640 & Peripheral & 206.50 & 142.79 & 239.45 & 143.16 & 160.98 \\
\hline 64680 & Celiac plexus & 178.30 & 144.25 & 184.90 & 136.20 & 160.98 \\
\hline 72265 & Myelography, lumbosacral, radiological S\&I & 202.11 & 202.11 & 201.38 & NA & 160.98 \\
\hline 72275 & Epidurography-radiological S\&I & 110.93 & 110.93 & 110.57 & NA & 234.19 \\
\hline 72285 & $\begin{array}{l}\text { Diskography, cervical or thoracic, radiological } \\
\text { S \& I }\end{array}$ & 384.44 & 326.93 & 384.44 & NA & 234.19 \\
\hline 72295 & Diskography L/S spine, radiological S \& I & 349.99 & 305.69 & 351.09 & NA & 234.19 \\
\hline 73542 & SI-joint arthrography-radiological S\&I & 107.64 & 107.64 & 107.64 & NA & 132.85 \\
\hline 76005 & Fluoroscopic guidance & 79.45 & 79.45 & 20.87 & NA & 120.73 \\
\hline
\end{tabular}

[i]. Information obtained from the November 2, 1999 Federal Register. [ii]. Information obtained from the November 2, 1999 Federal Register. [iii]. Information obtained from the July 17, 2000 Federal Register. [iv]. Information obtained from the July 17, 2000 Federal Register. 
in a later interim action in a program memorandum issued, HCFA delineated certain services with replacement codes for pain management procedures but refused to include new procedures based on various technical limitations (8). We have been told that these services, which include percutaneous lysis of adhesions, transforaminal epidural injections, cervical facet joint nerve blocks, and neurolytic blocks, simply are not available to Ambulatory Surgery center patients, pending the final implementation of the ASC final rule in April 2001. The Association of the Pain Management of Anesthesiologists (AOPMA), now known as the American Society of Interventional Pain Physicians (ASIPP) advised HCFA that the process would take approximately three years or so. Finally, following the numerous requests from the American Society of Interventional Pain Physicians and Congressional membership, HCFA has softened its position somewhat in that the statement that the new procedures will be considered at the time when final rule for outpatient Surgery centers may be issued in November 2000 and comments will be requested (18). While the new rules are pending, this situation creates various issues in billing and coding for interventional pain physicians as well as Ambulatory Surgery centers. Finally, physician payment policies both final rules for 2000 and proposed rules for $2001(4,5)$, and HCFA's acceptance of bilateral coding for procedures also creates some changes in billing and coding for interventional pain procedures in all settings. Table 1 shows the impact of these regulations on various interventional pain procedures.

Issues of correct coding and medical necessity and guidelines with regards to frequency and number of interventions, combination of blocks/interventions, and number per setting continue to remain the same $(1,19)$.

Various descriptors of interventional pain procedures commonly utilized and examples for many of the situations encountered in interventional pain practices are reviewed here. However, this review will only provide generally accepted practice patterns in a safe and ethical manner. Essentially, these illustrations and the information provide practical considerations for the use of interventional techniques in the management of chronic pain based on the current state of the art and signs of interventional pain management, rules and regulations. However, this article and its descriptions do not constitute practice management or legal advice. In addition, these guidelines also do not constitute inflexible treatment recommendations. It is expected that a provider will establish a plan of care on a case by case basis taking into account an individual patient's medical condition, personal needs, and preferences, and the physician's experience. Thus, based on individual patient's needs, treatments provided, experience of the physician, billing and coding staff, and rules and regulation of local Medicare carriers, and other payors, various types of practice patterns including billing and coding are warranted.

\section{FACET JOINT BLOCKS AND NEUROLYSIS}

\section{Procedure (CPT) Codes 2000 (3)}

- 64470 - Injection, anesthetic agent and/or steroid, paravertebral facet joint or facet joint nerve, cervical or thoracic, single level

- 64472 - Injection, anesthetic agent and/or steroid, paravertebral facet joint or facet joint nerve, cervical or thoracic, each additional level

64475 - Injection, anesthetic agent and/or steroid, paravertebral facet joint or facet joint nerve, lumbar or sacral, single level

64476 - Injection, anesthetic agent and/or steroid, paravertebral facet joint or facet joint nerve, lumbar or sacral, each additional level

- $\quad 64626$ - Destruction by neurolytic agent, paravertebral facet joint nerve; cervical or thoracic, single level

- 64627 - Destruction by neurolytic agent, paravertebral facet joint nerve; cervical or thoracic, each additional level

- 64622 - Destruction by neurolytic agent, paravertebral facet joint nerve; lumbar or sacral, single level

64623 - Destruction by neurolytic agent, paravertebral facet joint nerve; lumbar or sacral, each additional level

- 27096 - Injection procedure for sacroiliac joint, arthrography and/or anesthetic/steroid

- 73542 - Radiological examination, sacroiliac joint arthrography, radiological supervision and interpretation

HCFA continued to propose higher reimbursement for injection procedure for sacroiliac joint arthrography and/or anesthetic/steroid (CPT code 27096), as well as hip arthrography in a nonfacility setting, $\$ 434, \$ 355$ to $\$ 371$ consecutively. This probably is an error on the part of HCFA; however, at this point, we are unable to determine the future consequences if and when HCFA realizes that they may have been over-reimbursing for these codes. Similarly, CPT 73542 - radiological examination, sacroiliac joint arthrography, radiological supervision and interpretation is also considered as a radiology code and in- 
terventional pain physicians may have difficulty getting reimbursed for this procedure.

\section{Examples}

\section{i. Cervical facet joint injection, single level (C5/6 joint) \\ Physician \\ - $\quad 64470-\mathrm{C} / \mathrm{T}$ facet joint injection, single level \\ Surgery center \\ 76005 - Fluoroscopic guidance \\ Medicare \\ - $\quad$ Cervical facet joint injection procedures are not approved for surgery centers.} Other carriers

- $\quad 64470-\mathrm{C} / \mathrm{T}$ facet joint injection, single level

ii. Multiple cervical facet joint injections (C4/5 through C6/7)

Physician

- $\quad 64470-\mathrm{C} / \mathrm{T}$ facet joint injection, single level

- 64472-51 (two units) - C/T facet joint injection, each additional level

- 76005 - Fluoroscopic guidance

Surgery center

Medicare

- Cervical facet joint injection procedures Other carriers are not approved for surgery centers.

- $\quad 64470-\mathrm{C} / \mathrm{T}$ facet joint injection, single level

- $\quad 64472-51$ (two units) $-\mathrm{C} / \mathrm{T}$ facet joint injection, each additional level

iii. Bilateral cervical facet joint injections $(\mathrm{C} 4 / 5$ through C6/7)

\section{Physician}

Medicare

- 64470-50-C/T facet joint injection, single level (bilateral)

- $\quad 64472-\mathrm{C} / \mathrm{T}$ facet joint injection, each additional level (right)

- $\quad 64472-\mathrm{C} / \mathrm{T}$ facet joint injection, each additional level (left)

- 76005 - Fluoroscopic guidance

For carriers allowing two levels of bilateral coding

- 64470-50 - C/T facet joint injection, single level (bilateral)
- 64472-50-C/T facet joint injection, each additional level (bilateral)

- $\quad 76005$ - Fluoroscopic guidance

For carriers who do not permit bilateral coding

- $\quad 64470-\mathrm{C} / \mathrm{T}$ facet joint injection, single level

- $\quad 64472-51$ (two units) $-\mathrm{C} / \mathrm{T}$ facet joint injection, each additional level

Surgery center 76005 - Fluoroscopic guidance

Medicare

- Cervical facet joint nerve block procedures are not approved for surgery centers.

For carriers allowing two levels of bilateral coding

- 64470-50 - C/T facet joint injection, single level (bilateral)

- 64472-50-C/T facet joint injection, each additional level (bilateral)

For carriers who do not permit bilateral coding

- $\quad 64470-\mathrm{C} / \mathrm{T}$ facet joint injection, single level

- $64472-51$ (two units) - C/T facet joint injection, each additional level

iv. Cervical facet joint nerve blocks, single joint (C5/6 joint - C5 and C6 medial branch nerves)

Physician

- $\quad 64470-\mathrm{C} / \mathrm{T}$ facet joint nerve block, single level

- 76005 - Fluoroscopic guidance

Surgery center

Medicare

- Cervical facet joint nerve block procedures are not approved for surgery centers.

Other carriers

- $\quad 64470-\mathrm{C} / \mathrm{T}$ facet joint nerve block, single level

Cervical facet joint nerve block, multiple levels (C3/4 and $\mathrm{C} 4 / 5$ joints $-\mathrm{C} 3, \mathrm{C} 4$, and $\mathrm{C} 5$ medial branch nerves)

Physician

- $\quad 64470-\mathrm{C} / \mathrm{T}$ facet joint nerve block, single level

- $64472-$ C/T Facet joint nerve block, each additional level

- $\quad 76005$ - Fluoroscopic guidance 
Surgery center

Medicare

- Cervical facet joint nerve block procedures are not approved for surgery centers.

Other carriers

- $\quad 64470-\mathrm{C} / \mathrm{T}$ facet joint injection, single level

- $\quad 64472-\mathrm{C} / \mathrm{T}$ facet joint injection, each additional level

vi. Cervical facet joint nerve blocks, multiple levels (C3/4 through C6/7 joints - C3 through C7 medial branches)

Physician

- $64470-\mathrm{C} / \mathrm{T}$ facet joint nerve block, single level

- 64472-51 (two units) - C/T facet joint nerve block, each additional level

- $76005-$ Fluoroscopic guidance

Surgery center

Medicare

- $\quad$ Cervical facet joint nerve block procedures are not approved for surgery centers.

Other carriers

- $64470-\mathrm{C} / \mathrm{T}$ facet joint nerve block, single level

- 64472-51 (two units) - C/T facet joint nerve block, each additional level

vii. Bilateral cervical facet joint nerve blocks (C5/6 and $\mathrm{C} 6 / 7$ joints - $\mathrm{C} 5$ through $\mathrm{C} 7$ facet joint nerves or medial branches)

Physician

Medicare

- 64470-50 - C/T facet joint nerve block, single level (bilateral)

- $64472-\mathrm{C} / \mathrm{T}$ facet joint nerve block, each additional level (right)

- $64472-\mathrm{C} / \mathrm{T}$ facet joint nerve block, each additional level (left)

- $\quad 76005$ - Fluoroscopic guidance

For carriers allowing two levels of bilateral coding

- 64470-50-C/T facet joint nerve block, single level (bilateral)

- 64472-50-C/T facet joint nerve block, each additional level (bilateral)

- $\quad 76005$ - Fluoroscopic guidance
For carriers who do not permit bilateral coding

- $\quad 64470-\mathrm{C} / \mathrm{T}$ facet joint nerve block, single level

- 64472-51 (two units) - C/T facet joint nerve block, each additional level

Surgery center 76005 - Fluoroscopic guidance

Medicare

- Cervical facet joint nerve block procedures are not approved for surgery centers.

For carriers allowing two levels of bilateral coding

- 64470-50 - C/T facet joint injection, single level (bilateral)

- 64472-50 - C/T facet joint injection, each additional level (bilateral)

For carriers who do not permit bilateral coding

- $\quad 64470-\mathrm{C} / \mathrm{T}$ facet joint injection, single level

- $64472-51$ (two units) - C/T facet joint injection, each additional level

viii. Thoracic facet joint injection, single level (T5/6 joint)

Physician

- $\quad 64470-\mathrm{C} / \mathrm{T}$ facet joint injection, single level

- 76005 - Fluoroscopic guidance

Surgery center

Medicare

- Thoracic facet joint injection procedures are not approved for surgery centers.

Other carriers

- $\quad 64470-\mathrm{C} / \mathrm{T}$ facet joint injection, single level

ix. Multiple thoracic facet joint injections (T4/5 through T6/7)

Physician

- $\quad 64470-\mathrm{C} / \mathrm{T}$ facet joint injection, single level

- 64472-51 (two units) - C/T facet joint injection, each additional level

- 76005 - Fluoroscopic guidance

Surgery center

Thoracic facet joint injection procedures are not approved for surgery centers.

Other carriers

- $\quad 64470-\mathrm{C} / \mathrm{T}$ facet joint injection, single level 


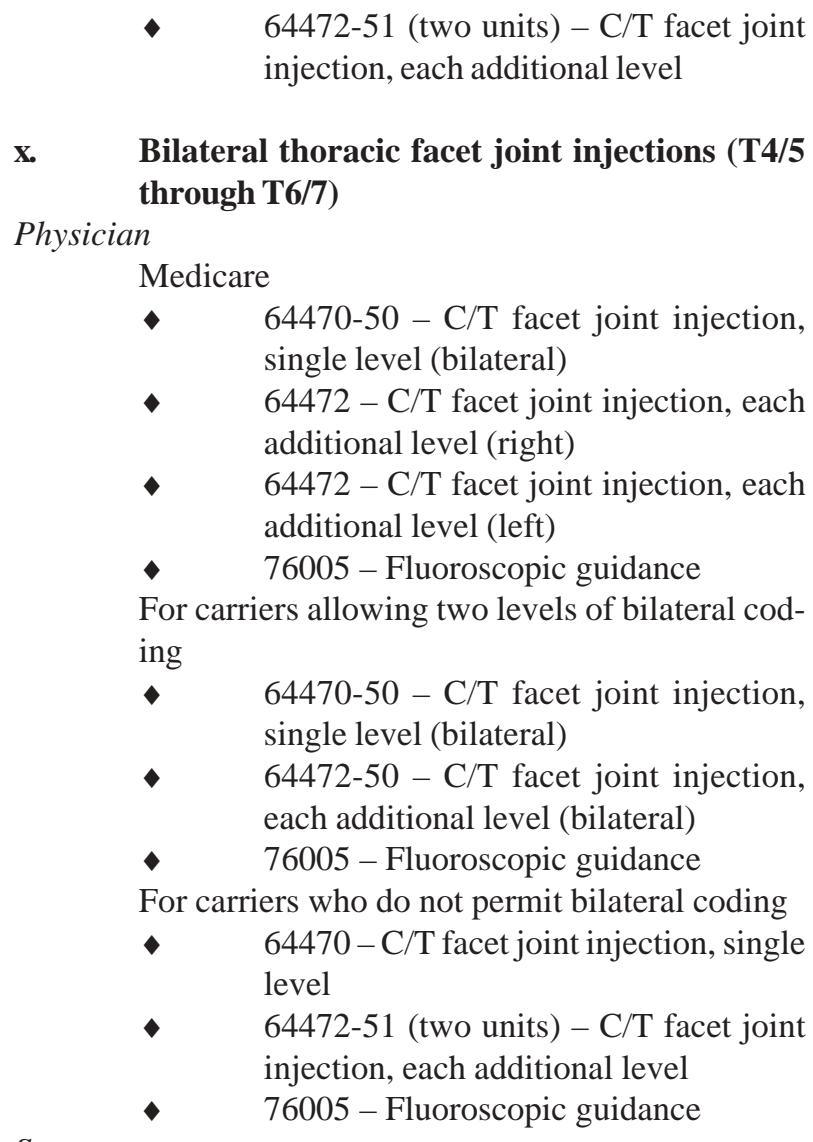

x. Bilateral thoracic facet joint injections (T4/5 Physician through T6/7)

Medicare

- 64470-50 - C/T facet joint injection, single level (bilateral)

- $\quad 64472-\mathrm{C} / \mathrm{T}$ facet joint injection, each additional level (right)

- $64472-\mathrm{C} / \mathrm{T}$ facet joint injection, each additional level (left)

- 76005 - Fluoroscopic guidance

For carriers allowing two levels of bilateral coding

- 64470-50 - C/T facet joint injection, single level (bilateral)
$64472-50-\mathrm{C} / \mathrm{T}$ facet joint injection, each additional level (bilateral)

- 76005 - Fluoroscopic guidance For carriers who do not permit bilateral coding

- $\quad 64470-\mathrm{C} / \mathrm{T}$ facet joint injection, single level

- 64472-51 (two units) - C/T facet joint injection, each additional level 76005 - Fluoroscopic guidance

Surgery center

Medicare

- Thoracic facet joint injection procedures are not approved for surgery centers.

For carriers allowing two levels of bilateral coding

- 64470-50 - C/T facet joint injection, single level (bilateral)

- 64472-50 - C/T facet joint injection, each additional level (bilateral)

For carriers who do not permit bilateral coding

- $\quad 64470-\mathrm{C} / \mathrm{T}$ facet joint injection, single level

- 64472-51 (two units) - C/T facet joint injection, each additional level

xi. Thoracic facet joint nerve blocks, single joint Physician ( $T 5 / 6$ joint $-\mathrm{T} 5$ and $\mathrm{T} 6$ medial branch nerves)

- $64470-\mathrm{C} / \mathrm{T}$ facet joint nerve block, single level

• 76005 - Fluoroscopic guidance
Surgery center

Medicare

- Thoracic facet joint nerve block procedures are not approved for surgery centers.

Other carriers

- $64470-\mathrm{C} / \mathrm{T}$ facet joint nerve block, single level

xii. Thoracic facet joint nerve blocks, multiple levels (T3/4 through T6/7 joints - T3 through T7 medial branches)

Physician

- $64470-\mathrm{C} / \mathrm{T}$ facet joint nerve block, single level

- 64472-51 (two units) - C/T facet joint nerve block, each additional level

- 76005 - Fluoroscopic guidance

Surgery center

Medicare

- Thoracic facet joint nerve block procedures are not approved for surgery centers.

Other carriers

- $64470-\mathrm{C} / \mathrm{T}$ facet joint nerve block, single level

- 64472-51 (two units) - C/T facet joint nerve block, each additional level

xiii. Bilateral thoracic facet joint nerve blocks (T5/6 and $\mathrm{T} 6 / 7$ joints $-\mathrm{T} 5$ through $\mathrm{T} 7$ facet joint nerves or medial branch nerves)

Physician

- 64470-50 - C/T facet joint nerve block, single level (bilateral)

- $\quad 64472-\mathrm{C} / \mathrm{T}$ facet joint nerve block, single level (right)

- $64472-\mathrm{C} / \mathrm{T}$ facet joint nerve block, single level (left)

- $\quad 76005$ - Fluoroscopic guidance

For carriers allowing two levels of bilateral coding

- 64470-50-C/T facet joint nerve block, single level (bilateral)

- 64472-50-C/T facet joint nerve block, each additional level (bilateral)

- 76005 - Fluoroscopic guidance

For carriers who do not permit bilateral coding

- $64470-\mathrm{C} / \mathrm{T}$ facet joint nerve block, single level 


\section{- 64472-51 (two units) - C/T facet joint nerve block, subsequent levels \\ - 76005 - Fluoroscopic guidance \\ Surgery center \\ Medicare \\ - Thoracic facet joint nerve block proce- dures are not approved for surgery cen- ters. \\ For carriers allowing two levels of bilateral cod- ing \\ - 64470-50 - C/T facet joint injection, single level (bilateral) \\ - 64472-50 - C/T facet joint injection, each additional level (bilateral) \\ For carriers who do not permit bilateral coding \\ - $\quad 64470-\mathrm{C} / \mathrm{T}$ facet joint injection, single level \\ - 64472-51 (two units) - C/T facet joint injection, each additional level}

xiv. Lumbosacral facet joint injection, single level (L3/4 joint)

Physician

- $\quad 64475-\mathrm{L} / \mathrm{S}$ facet joint injection, single level

Surgery center

- $64475-\mathrm{L} / \mathrm{S}$ facet joint injection, single level

xv. Lumbosacral facet joint injections, multiple levels $(\mathbf{L} 2 / 3-\mathbf{L} 5 / \mathbf{S 1})$

Physician

- $\quad 64475-\mathrm{L} / \mathrm{S}$ facet joint injection, single level

- 64476-51 (two units) - L/S facet joint injection, each additional level

- $\quad 76005$ - Fluoroscopic guidance

Surgery center

- $\quad 64475-\mathrm{L} / \mathrm{S}$ facet joint injection, single level

- 64476-51 (two units) - L/S facet joint injection, each additional level

xvi. Bilateral lumbosacral facet joint injections Physician (L3/4 and L4/5 joints)

Medicare

- 64475-50 - L/S facet joint injection, single level (bilateral)

- $\quad 64476-$ L/S facet joint injection, each
additional level (right)
- $64476-\mathrm{L} / \mathrm{S}$ facet joint injection, each additional level (left)
- $\quad 76005$-Fluoroscopic guidance

For carriers allowing two levels of bilateral coding then use

- 64475-50 - L/S facet joint nerve block, single level (bilateral)

- 64476-50 - L/S facet joint nerve block, each additional level (bilateral)

- $\quad 76005$ - Fluoroscopic guidance

For carriers who do not permit bilateral coding

- $64475-$ L/S facet joint nerve block, single level (bilateral)

- 64476-51(two units) - L/S facet joint nerve block, each additional level (bilateral)

Surgery center

76005 - Fluoroscopic guidance

Medicare

- 64475-50 - L/S facet joint injection, single level (bilateral)

- $\quad 64476-$ L/S facet joint injection, each additional level (right)

- $\quad 64476-$ L/S facet joint injection, each additional level (left)

For carriers allowing two levels of bilateral coding

- 64475-50 - L/S facet joint injection, single level (bilateral)

- 64476-50 - L/S facet joint injection, each additional level (bilateral)

For carriers who do not permit bilateral coding

- $\quad 64475-\mathrm{L} / \mathrm{S}$ facet joint injection, single level

- 64476-51 (two units) - L/S facet joint injection, each additional level

xvii. Lumbar facet joint nerve blocks, single joint (L3/ 4 joint $-\mathrm{L} 2$ and L3 medial branch nerves or facet joint nerves)

Physician

- $\quad 64475-$ L/S facet joint nerve block, single level

Surgery center

76005 - Fluoroscopic guidance

64475 - L/S facet joint injection, single level 
xviii. Multiple lumbar facet joint nerve blocks ( $L 4 / 5$ and L5/S1 joints - L3 and L4 medial branch nerves and $\mathrm{L} 5$ dorsal ramus)

Physician

- $64475-\mathrm{L} / \mathrm{S}$ facet joint nerve block, single level

- $\quad 64476-\mathrm{L} / \mathrm{S}$ facet joint nerve block, each additional level

Surgery center

- $64475-\mathrm{L} / \mathrm{S}$ facet joint nerve block, single level

- 64476-51 - L/S facet joint nerve block, each additional level

xix. Bilateral multiple lumbar facet joint nerve blocks (L3/4 through L5/S1 joints - L2 through L4 medial branch nerves and $\mathrm{L} 5$ dorsal ramus)

\section{Physician}

Medicare

- 64475-50 - L/S facet joint nerve block, single level (bilateral)

- $\quad 64476-\mathrm{L} / \mathrm{S}$ facet joint nerve block, each additional level (right)

- $\quad 64476-\mathrm{L} / \mathrm{S}$ facet joint nerve block, each additional level (left)

- $\quad 76005$ - Fluoroscopic guidance

For carriers allowing two levels of bilateral coding

- 64475-50 - L/S facet joint nerve block, single level (bilateral)

- 64476-50 - L/S facet joint nerve block, each additional level (bilateral)

- $\quad 76005$ - Fluoroscopic guidance

For carriers who do not permit bilateral coding

- $64475-$ L/S facet joint nerve block, single level

- 64476-51 (two units) - L/S facet joint nerve block, each additional level

- $\quad 76005-$ Fluoroscopic guidance

\section{Surgery center}

Medicare

- 64475-50 - L/S facet joint nerve block, single level (bilateral)

- 64476-51 - L/S facet joint nerve block, each additional level (right)

- 64476-51 - L/S facet joint nerve block, each additional level (left)

For carriers allowing two levels of bilateral coding

64475-50 - L/S facet joint nerve block, single level (bilateral)
- 64476-50 - L/S facet joint nerve block each additional level (bilateral)

For carriers who do not permit bilateral coding

- $64475-$ L/S facet joint nerve block, single level

- 64476-51 (two units) - L/S facet joint nerve block, each additional level

xx. Cervical paravertebral facet joint nerve neurolysis - single joint ( $\mathrm{C5} / 6$ joint $-\mathrm{C} 5$ and $\mathrm{C} 6$ medial branch nerves)

Physician

- $64626-\mathrm{C} / \mathrm{T}$ facet neurolysis, single level

- 76005 - Fluoroscopic guidance

Surgery center

Medicare

- Cervical paravertebral facet joint nerve neurolysis procedures are not approved for surgery centers.

Other carriers

- $64626-\mathrm{C} / \mathrm{T}$ facet neurolysis, single level

xxi. Multiple cervical paravertebral facet joint neurolysis $(\mathrm{C} 4 / 5$ through $\mathrm{C} 6 / 7$ joints $-\mathrm{C} 4$ through C7 medial branches)

Physician

- $\quad 64626-\mathrm{C} / \mathrm{T}$ facet joint neurolysis, single level

- 64627-51 (two units) - C/T facet joint neurolysis, each additional level

- 76005 - Fluoroscopic guidance

Surgery center

Medicare

- Cervical paravertebral facet joint neurolysis procedures are not approved for surgery centers.

Other carriers

- $\quad 64626-\mathrm{C} / \mathrm{T}$ facet joint neurolysis, single level

- 64627-51 (two units) - C/T facet joint neurolysis, each additional level

xxii. Bilateral thoracic facet joint nerve neurolysis (T4/5 through T6/7 joints - T4 through T7 medial branches)

Physician

Medicare

- 64626-50 - C/T facet joint nerve neurolysis, single level (bilateral) 
- $\quad 64627-\mathrm{C} / \mathrm{T}$ facet joint nerve neurolysis, each additional level (right)

- $\quad 64627-\mathrm{C} / \mathrm{T}$ facet joint nerve neurolysis, each additional level (left)

- 76005 - Fluoroscopic guidance

For carriers allowing two levels of bilateral coding

- 64626-50 - C/T facet joint nerve neurolysis, single level (bilateral)

- 64627-50 - C/T facet joint nerve neurolysis, each additional level (bilateral)

- 76005 - Fluoroscopic guidance

For carriers who do not permit bilateral coding

- $\quad 64626-\mathrm{C} / \mathrm{T}$ facet joint nerve neurolysis, single level

- 64627-51 (two units) - C/T facet joint nerve neurolysis, each additional level

Surgery center

76005 - Fluoroscopic guidance

Medicare

- Thoracic facet joint nerve neurolytic procedures are not approved for surgery centers.

For carriers allowing two levels of bilateral coding

- 64626-50 - C/T facet joint nerve neurolysis, single level (bilateral)

- 64627-50 - C/T facet joint nerve neurolysis, each additional level (bilateral)

For carriers who do not permit bilateral coding

- $\quad 64626-\mathrm{C} / \mathrm{T}$ facet joint nerve neurolysis, single level

- 64627-51 (two units) - C/T facet joint nerve neurolysis, each additional level

xxiii. Thoracic paravertebral facet joint nerve neurolysis - single joint (T5/6 joint - T5 and T6 medial branch nerves)

Physician

- 64626-C/T paravertebral facet joint neurolysis, single level

- 76005 - Fluoroscopic guidance

Surgery center

Medicare

- Thoracic paravertebral facet joint nerve neurolysis procedures are not approved for surgery centers.

Other carriers

- $\quad 64626-\mathrm{C} / \mathrm{T}$ paravertebral facet joint neurolysis, single level xxiv. Multiple thoracic paravertebral facet joint neurolysis (T4/5 through T6/7 joints - T4 through T7 medial branches)

Physician

- $\quad 64626-\mathrm{C} / \mathrm{T}$ paravertebral facet joint neurolysis, single level

- 64627-51 (two units) - C/T paravertebral facet joint neurolysis, each additional level

- 76005 - Fluoroscopic guidance

\section{Surgery center}

- Thoracic paravertebral facet joint neurolytic procedures are not approved for surgery centers.

Other carriers
- $64626-\mathrm{C} / \mathrm{T}$ paravertebral facet joint neurolysis, single level
- 64627-51 (two units) - C/T paraverte- bral facet joint neurolysis, each additional level

XXv. Lumbar paravertebral facet joint neurolysis single joint (L4/5 joint - L3 and L4 medial branch nerves)

Physician

- $\quad 64622-\mathrm{L} / \mathrm{S}$, paravertebral facet joint neurolysis, single level

- 76005 - Fluoroscopic guidance

Surgery center

- $64622-\mathrm{L} / \mathrm{S}$, paravertebral facet joint neurolysis, single level

xxvi. Multiple lumbar paravertebral facet joint neurolysis (L3/4 through L5/S1 joints L2 through L4 medial branch nerves and L5 dorsal ramus)

Physician

- $64622-$ L/S paravertebral facet joint neurolysis, single level

- 64623-51 (two units) - L/S paravertebral facet joint neurolysis, each additional level

• 76005 - Fluoroscopic guidance

Surgery center

- 64622 - L/S paravertebral facet joint neurolysis, single level

- 64623-51 (two units) - L/S paravertebral facet joint neurolysis, each additional level 
xxvii. Bilateral lumbar facet joint neurolysis (L3/4 through L5/S1 joints - L2 through L4 medial branch nerves and $\mathrm{L} 5$ dorsal ramus)

Physician

Medicare

- $\quad 64622-50-\mathrm{L} / \mathrm{S}$ paravertebral facet joint neurolysis, single level (bilateral)

- $\quad 64623-\mathrm{L} / \mathrm{S}$ paravertebral facet joint neurolysis, each additional level (right)

- $64623-\mathrm{L} / \mathrm{S}$ paravertebral facet joint neurolysis, each additional level (left)

- $\quad 76005$ - Fluoroscopic guidance

For carriers allowing two levels of bilateral coding

- 64622-50 - L/S facet joint nerve neurolysis, single level (bilateral)

- 64623-50 - L/S facet joint nerve neurolysis, each additional level (bilateral)

- $\quad 76005$ - Fluoroscopic guidance

For carriers who do not permit bilateral coding

- $64622-\mathrm{L} / \mathrm{S}$ facet joint nerve neurolysis, single level

- 64623-51 (two units) - L/S facet joint nerve neurolysis, each additional level

Surgery center

76005 - Fluoroscopic guidance

Medicare

- 64622-50 - L/S paravertebral facet joint neurolysis, single level (bilateral)

- 64623-51 - L/S paravertebral facet joint neurolysis, each additional level (right)

- 64623-51-L/S paravertebral facet joint neurolysis, each additional level (left)

For carriers allowing two levels of bilateral coding

- 64622-50 - L/S facet joint nerve neurolysis, single level (bilateral)

- 64623-50 - L/S facet joint nerve neurolysis, each additional level (bilateral)

For carriers who do not permit bilateral coding

- $64622-\mathrm{L} / \mathrm{S}$ facet joint nerve neurolysis, single level

- 64623-51 (two units) - L/S facet joint nerve neurolysis, each additional level

\section{EPIDURAL INJECTIONS}

\section{Procedure (CPT) 2000 Codes (3)}

- $\quad 62310$ - Injection, single (not via indwelling catheter), not including neurolytic substances, with or without contrast (for either localization or epidurography), of diagnostic or therapeutic substance(s) (including anesthetic, antispasmodic, opioid, steroid, other solution), epidural or subarachnoid; cervical or thoracic

62311 - Injection, single (not via indwelling catheter), not including neurolytic substances, with or without contrast (for either localization or epidurography), of diagnostic or therapeutic substance(s) (including anesthetic, antispasmodic, opioid, steroid, other solution), epidural or subarachnoid; lumbar, sacral (caudal)

- 62318 - Injection, including catheter placement, continuous infusion or intermittent bolus, not including neurolytic substances, with or without contrast (for either localization or epidurography), of diagnostic or therapeutic substance(s) (including anesthetic, antispasmodic, opioid, steroid, other solution), epidural or subarachnoid; cervical or thoracic 62319 - Injection, including catheter placement, continuous infusion or intermittent bolus, not including neurolytic substances, with or without contrast (for either localization or epidurography), of diagnostic or therapeutic substance(s) (including anesthetic, antispasmodic, opioid, steroid, other solution), epidural or subarachnoid; lumbar, sacral (caudal)

- 64479 - Injection, anesthetic agent and/ or steroid, transforaminal epidural; cervical or thoracic, single level

- 64480 - Injection, anesthetic agent and/ or steroid, transforaminal epidural; cervical or thoracic, each additional level 64483 - Injection, anesthetic agent and/ or steroid, transforaminal epidural; lumbar or sacral, single level

- 64484 - Injection, anesthetic agent and/ or steroid, transforaminal epidural; lumbar or sacral, each additional level

- $\quad 72275$ - Epidurography, radiological supervision and interpretation

- 76005 - Fluoroscopic guidance and localization of needle or catheter tip for spine or paraspinous diagnostic or therapeutic procedures 
The new developments include reduction or elimination of reimbursement for CPT 76005 - Fluoroscopic guidance and localization of needle or catheter tip or paraspinous diagnostic or therapeutic procedures. In addition, carriers across the country have been refusing payment for CPT 72275 - Epidurography, radiological supervision and interpretation stating that this is a radiology code and there are no specific indications for this procedure. Therefore, interventional pain physicians will not be reimbursed for this code.

\section{Examples}

i. Cervical interlaminar epidural injection - withPhysician out fluoroscopy

$\begin{array}{cc}\bullet & 62310-\mathrm{C} / \mathrm{T} \text { epidural } \\ \text { Surgery center } & \\ \bullet & 62310-\mathrm{C} / \mathrm{T} \text { epidural }\end{array}$

ii. Cervical interlaminar epidural injection - with fluoroscopy

Physician

$62310-\mathrm{C} / \mathrm{T}$ epidural
$76005-$ Fluoroscopic guidance

Surgery center

- $\quad 62310-\mathrm{C} / \mathrm{T}$ epidural

iii. Thoracic interlaminar epidural injection - withPhysician out fluoroscopy

$\begin{array}{cc}\bullet & 62310-\mathrm{C} / \mathrm{T} \text { epidural } \\ \text { Surgery center } & \\ \bullet & 62310-\mathrm{C} / \mathrm{T} \text { epidural }\end{array}$

iv. Thoracic interlaminar epidural injection - with fluoroscopy

Physician

- $\quad 62310-\mathrm{C} / \mathrm{T}$ epidural

- 76005 - Fluoroscopic guidance

Surgery center

62310- C/T epidural

v. Lumbar interlaminar epidural injection - without fluoroscopy

Physician

- $\quad 62311-$ L/S epidural

Surgery center

- 62311-Lumbar epidural vi. Lumbar interlaminar epidural - with fluoroscopy Physician

$62311-\mathrm{L} / \mathrm{S}$ epidural
$76005-$ Fluoroscopic guidance

Surgery center

62311-Lumbar epidural

vii. Caudal epidural injection - without fluoroscopy

Physician

62311-L/S epidural

- 76005 - Fluoroscopic guidance

Surgery center

- 62311- Lumbar/caudal epidural

viii. Caudal epidural injection - with fluoroscopy

Physician

$\begin{array}{cl}\bullet & 62311-\text { L/S epidural } \\ \bullet & 76005-\text { Fluoroscopic guidance } \\ \text { Surgery center } & \\ \bullet & 62311-\text { Lumbar/caudal epidural }\end{array}$

ix. Cervical transforaminal epidural injection, single level (C5 spinal nerve, C4/5 foramen)

Physician

- 64479 - C/T transforaminal epidural, single level

Surgery center

76005 - Fluoroscopic guidance

Medicare

Cervical transforaminal epidural injection procedures are not approved for surgery centers.

Other carriers

- 64479 - C/T transforaminal epidural, single level (C5)

x. Cervical transforaminal epidural injection, multiple levels (C6 and C7 spinal nerves)

Physician

- $64479-\mathrm{C} / \mathrm{T}$ transforaminal epidural, single level

- 64480 - C/T transforaminal epidural, each additional level

- 76005 - Fluoroscopic guidance

Surgery center

Medicare

- Cervical transforaminal epidural injection procedures are not approved for surgery centers.

Other carriers

64479 - C/T transforaminal epidural, single level 
- 64480 - C/T transforaminal epidural, each additional level

xi. Thoracic transforaminal or selective epidural injection, single level (T5 spinal nerve, T4/5 foramen)

Physician

- $64479-\mathrm{C} / \mathrm{T}$, transforaminal epidural, single level

Surgery center

76005 - Fluoroscopic guidance

Medicare

- Thoracic transforaminal or selective epidural injection procedures are not apOther carriers proved for surgery centers.

- $64479-\mathrm{C} / \mathrm{T}$, transforaminal epidural, single level

xii. Thoracic transforaminal or selective epidural inPhysician jection, multiple levels (T6 and T7 spinal nerves)

- $64479-\mathrm{C} / \mathrm{T}$, single level

- $64480-\mathrm{C} / \mathrm{T}$ transforaminal epidural, each additional level

Surgery center

76005 - Fluoroscopic guidance

Medicare

Thoracic transforaminal or selective epidural injection procedures are not approved for surgery centers.

Other carriers

$64479-\mathrm{C} / \mathrm{T}$, single level
$64480-\mathrm{C} / \mathrm{T}$ transforaminal epidural, each additional level

xiii. Bilateral thoracic transforaminal or selective epidural injections (T5, T6, and T7 spinal nerves) Physician

Medicare

- 64479-50 - CT transforaminal epidural, single level (bilateral)

- 64480 - C/T transforaminal epidural, each additional level (right)

- 64480 - C/T transforaminal epidural, each additional level (left)

- 76005 - Fluoroscopic guidance

For carriers allowing two levels of bilateral coding

- 64479-50 - C/T transforaminal epidural, single level (bilateral)
- 64480-50 - C/T transforaminal epidural, each additional level (bilateral)

- 76005 - Fluoroscopic guidance For carriers who do not permit bilateral coding

- 64479 - C/T transforaminal epidural, single level

- 64480-51 (two units) - C/T transforaminal epidural, each additional level

Surgery center

76005 - Fluoroscopic guidance

Medicare

- Thoracic transforaminal or selective epidural injections procedures are not Other carriers approved for surgery centers.

For carriers allowing two levels of bilateral coding

- 64479-50 - C/T transforaminal epidural, single level (bilateral)

- 64480-50 - C/T transforaminal epidural, each additional level (bilateral)

For carriers who do not permit bilateral coding

- 64479 - C/T transforaminal epidural, single level

- 64480-51 (two units) - C/T transforaminal epidural, each additional level

xiv. Lumbar transforaminal or selective epidural injection (L5 spinal nerve)

Physician

- 64483 - L/S transforaminal epidural, single level

Surgery center

76005 - Fluoroscopic guidance

Medicare

- Lumbar transforaminal or selective epidural injection procedures are not apOther carriers proved for surgery centers.

- 64483 - L/S transforaminal epidural, single level

xv. Lumbar transforaminal or selective epidural injection, multiple levels (L4, L5, and S1 spinal nerves)

Physician

64483 - L/S transforaminal epidural, single level

- 64484-51 (two units) - L/S transforaminal epidural, each additional level

- 76005 - Fluoroscopic guidance 
Surgery center

Medicare

- Lumbar transforaminal or selective epidural injection codes are not approved procedures for surgery centers.

\section{Other carriers}

- $64483-\mathrm{L} / \mathrm{S}$ transforaminal epidural, single level

- 64484-51 (two units) - L/S transforaminal epidural, each additional level

\section{PERCUTANEOUS LYSIS OF EPIDURAL ADHESIONS}

\section{Procedure (CPT) Code (3)}

- $\quad 62263$ - Percutaneous lysis of epidural adhesions using solution injection (eg, hypertonic saline, enzyme) or mechanical means (eg, spring-wire catheter) including radiologic localization (includes contrast when administered) xvi. Bilateral lumbar transforaminal or selective epidural injections (L5, S1)

\section{Physician}

Medicare

- 64483-50 - L/S transforaminal epidural, single level (bilateral)

- 64484 - L/S transforaminal epidural, each additional level (right)

- 64484 - L/S transforaminal epidural, each additional level (left)

- 76005 - Fluoroscopic guidance

For carriers allowing two levels of bilateral coding

- 64483-50 - L/S transforaminal epidural, single level (bilateral)

- 64484-50 - L/S transforaminal epidural, each additional level (bilateral)

- $\quad 76005$ - Fluoroscopic guidance

For carriers who do not permit bilateral coding

- 64483 - L/S transforaminalepidural, single level

- 64484-51 (two units) - L/S transforaminal epidural, each additional level

- 76005 - Fluoroscopic guidance

Surgery center

Medicare

- Lumbar transforaminal or selective epidural injection procedures are not approved for surgery centers.

For carriers allowing two levels of bilateral coding

- 64483-50 - L/S transforaminal epidural, single level (bilateral)

- 64484-50 - L/S transforaminal epidural, each additional level (bilateral)

For carriers who do not permit bilateral coding

- 64483 - L/S transforaminal epidural, single level

- 64484-51 (two units) - L/S transforaminal epidural, each additional level

\section{Example}

Percutaneous lysis of adhesions utilizing Racz catheter Physician

- $62263-$ Percutaneous lysis of epidural adhesions

Surgery center

Medicare

- Percutaneous lysis of adhesions epidural adhesions is not approved for surgery centers.

Other carriers

- 62263 - Percutaneous lysis of epidural adhesions

\section{DISCOGRAPHY AND ANNULOPLASTY}

\section{Procedure (CPT) Codes (3)}

62290 - Injection procedure for discography, each level; lumbar

62291 - Injection procedure for discography, each level; cervical or thoracic

72285 - Diskography, cervical or thoracic, radiological supervision and interpretation

72295 - Diskography, lumbar, radiological supervision and interpretation

- No CPT codes are available for intradiscal thermal annuloplasty. The following code is used with revised description:

62287 - Aspiration or decompression procedure, percutaneous, of nucleus pulposus of intervertebral disk, any method, single or multiple levels, lumbar (eg, manual or automated percutaneous diskectomy, percutaneous laser diskectomy)

Once again, similar to epidurography and sacroiliac joint discography codes with radiological supervision and interpretation, codes 72285 and 72295 continue to have difficulty getting reimbursed. In addition, intradiscal electri- 
cal thermal annuloplasty also has elicited significant controversy. The American Medical Association initially issued an opinion that CPT 62287 (aspiration or decompression procedure) was the appropriate code to utilize. However, later on, the AMA advised to use an unlisted procedure code for the nervous system, namely CPT 22899, and yet another unused code also emerged, i.e. CPT 64999. Further, recent advice was extended to the use of CPT 62292 (injection procedure for chemonucleolysis, including diskography, intervertebral disk, single or multiple levels, lumbar). However, none of these codes are approved for Surgery centers at this time. Recently, the AMA has been considering revision of CPT 62287 so that the definition of the code would incorporate intradiscal thermal annuloplasty. Thus, CPT 62287, may be considered as appropriate for continued to use to describe IDET. However, caution must be exercised prior to utilizing this code on any patient. All specialists performing these procedures are requested to obtain pre-approvals with the explicit understanding of the insurer of the procedure to be performed and the code to be utilized. In addition, providers should also obtain in writing the clarification of use of the insurers that they do indeed understand the nature of the procedure i.e. intradiscal thermal annuloplasty and the CPT code 62287 i.e. aspiration or decompression procedure, percutaneous, of nucleus pulposus of intervertebral disk, any method, single or multiple levels is being used.

\section{$\operatorname{Example}(s)$}

i. Cervical discography - multiple levels (C4/5, C5/6)

\section{Physician}

- $62291-\mathrm{C} / \mathrm{T}$ discography, each level

62291-51-C/T discography, each level

- $72285-\mathrm{C} / \mathrm{T}$ discography, radiological

Surgery center supervision and interpretation

Medicare

- Cervical discography procedures are not Other carriers approved for surgery centers.

$62291-\mathrm{C} / \mathrm{T}$ discography, each level
$62291-51-\mathrm{C} / \mathrm{T}$ discography, each level

ii. Thoracic discography - multiple levels (T4/5, T5/6)

Physician

$62291-\mathrm{C} / \mathrm{T}$ discography, each level

- 62291-51-C/T discography, each level
- $\quad 72285-\mathrm{C} / \mathrm{T}$ discography, radiological supervision and interpretation

Surgery center

Medicare

- Thoracic discography procedures are Other carriers not approved for surgery centers.

- $62291-\mathrm{C} / \mathrm{T}$ discography, each level

- 62291-51-C/T discography, each level

iii. Lumbar discography - multiple levels (L3/4, L4/5, L5/S1)

Physician

- 62290-Lumbar discography, each level

- 62290-51 - Lumbar discography, each level

- $\quad 72295$ - Lumbar discography, radiological supervision and interpretation

Surgery center

Medicare

- Lumbar discography procedures are not approved for surgery centers.

Other carriers

- 62290 - Lumbar discography, each level

- 62290-51 - Lumbar discography, each level

iv. Intradiscal electric thermal annuloplasty, single or multiple levels

Physician

- 62287 - Aspiration or decompression procedure, percutaneous, of nucleus pulposus of intervertebral disk, any method, single or multiple levels; or

- 22899 - Unlisted, spine procedure; or

- 64999 - unlisted, spine procedure

Surgery center

Medicare

- Intradiscal electrical thermal annuloplasty procedures are not approved for surgery centers.

Other carriers

- 62287 - Aspiration or decompression procedure, percutaneous, of nucleus pulposus of intervertebral disk, any method, single or multiple levels; or

- 22899 - Unlisted, spine procedure; or

- 64999 - unlisted, spine procedure 


\section{MULTIPLE REGIONS AND/OR COMBINATIONS}

Due to various regulations in existence at the present time, when interventional procedures are performed in multiple regions and/or combinations, thus could be utilized for the advantage of the physician in certain cases. It is common to perform blocks in multiple regions in interventional pain practices as it has been shown that involvement of multiple regions in chronic pain patients is more prevalent than assumed (20). It has been shown that in patients suffering with chronic low back pain presenting to interventional pain practice, patients who had three regions being affected were $33 \%$ followed by two regions with $46 \%$ and the remaining $21 \%$ suffered with pain in only one region. In addition, treating multiple regions at one time is not only beneficial to the patient, it is also cost and time saving for the insurer, the facility, and time saving for physician and staff. The most common complication in including multiple regions into the treatment regimen at one time steroid toxicity. However, this has been shown to be negligible when therapy was carried out with a low dose or no steroids (20). Creative billing will only create multiple problems for interventional pain practitioners all across the country as insurers universally blame interventional pain physicians across the board citing examples of 24 charges on one patient, etc. The guidelines here indicate a limitation of 4 procedures or line items for management of a single region and a 5 for multiple regions. Thus, if a bilateral procedure is performed at two levels, that it will constitute 4 procedures, such as needle placement, epidurography, radiological interpretation and supervision, etc. are also considered as a line item. Most carriers of Medicare do not reimburse more than 5 line items, codes, or procedures at one time per patient. In addition, some insurers do not reimburse if a procedure code is repeated following the primary charge as an increase in the number of units. Considering the present situation with the proposed ASC ruling, higher reimbursement may be possible, within legal and ethical parameters without using creative billing techniques, for procedures which are performed in a facility setting which is the same as if it is performed in a nonfacility setting. It may be worthwhile for physicians to pay proper attention to billing and coding practices when multiple regions or combinations are utilized in managing these patients. Once again, these practices should be performed within the limits of the guidelines following the rules, regulations and ethical practices. Benefits may not be realized to the fullest extent if the physician has an agreement with the surgery center to reimburse the surgery center for the facility portion of the reimbursement for nonapproved procedures which is nonfacility total reimbursement minus facility reimbursement; but, still will yield higher reimbursement and is the right thing to do.

\section{Examples}

i. Multiple cervical facet joint nerve blocks, (C3/4C5 6 joints - C3-6 medial branch nerves) and multiple lumbar facet joint nerve block, (L3/4-L5/S1 joints L2-L4 medial branches and L5 dorsal ramus)

Physician

- $\quad 64470-\mathrm{C} / \mathrm{T}$ facet joint nerve block, single level

- $64472-\mathrm{C} / \mathrm{T}$ facet joint nerve block, each additional level

- $64475-\mathrm{L} / \mathrm{S}$ facet joint nerve block, single level

- $\quad 64476-\mathrm{L} / \mathrm{S}$ facet joint nerve block, each additional level

- 76005 - Fluoroscopic guidance

Surgery center

Medicare

- Multiple cervical facet joint nerve blocks are not approved procedures for surgery centers. Hence, the charges for the facility are limited to lumbar facet joint nerve block only:

- $64475-$ L/S facet joint nerve block, single level

- 64476-51 (two units) - L/S facet joint nerve block, each additional level

Other Carriers

- $\quad 64470-\mathrm{C} / \mathrm{T}$ facet joint nerve block, single level

- 64472-51-C/T facet joint nerve block, each additional level

- 64475-51 - L/S facet joint nerve block, single level

- 64476-51 - L/S facet joint nerve block, each additional level (may or may not be used considering the charge level of the facility)

ii. Caudal epidural and multiple cervical facet joint nerve blocks (C3/4-C5/6 joints - C3-6 medial branch nerves)

Physician

- $\quad 64470-$ CT facet joint nerve block, single level

- $\quad 64472-\mathrm{C} / \mathrm{T}$ facet joint nerve block, each additional level

62311-51-L/S epidural 
- 76005 -Fluoroscopic guidance (may or may not be used)

Surgery center

Medicare

- Cervical facet joint nerve block procedures are not approved for surgery centers.

- 62311-Lumbosacral epidural

Other Carriers

$62311-\mathrm{L} / \mathrm{S}$ epidural

- 64470-51 - CT facet joint nerve block, single level

- 64472-51-C/T facet joint nerve block, each additional level

iii. Cervical epidural and multiple lumbar facet joint nerve blocks

Physician

- $62310-$ Cervical epidural

- 64475-51 - L/S facet joint nerve block, single level

- $\quad 64476-\mathrm{L} / \mathrm{S}$ facet joint nerve block, each additional level; or

- $64476-51$ (two units) L/S facet joint nerve block, each additional level

- 76005 - Fluoroscopic guidance

Surgery center

- $62311-\mathrm{L} / \mathrm{S}$ epidural

- 64470-51 - CT facet joint nerve block, single level

- 64472-51-C/T facet joint nerve block, each additional level

iv. Multiple cervical transforaminal epidurals and multiple lumbar facet joint nerve blocks

Physician

- 64479 -Cervical transforaminal, single level

- $\quad 64481$ - Cervical transforaminal, each additional level

- $64475-$ L/S facet joint nerve block, single level

- $\quad 64476-\mathrm{L} / \mathrm{S}$ facet joint nerve block, each additional level

- 76005 - Fluoroscopic guidance

Surgery center

Medicare

- Cervical transforaminal injections are not approved for surgery centers.
- $\quad 64475-\mathrm{L} / \mathrm{S}$ facet joint nerve block, single level

- 64476-51 (two units) - L/S facet joint nerve block, each additional level (two units may be billed if a total of at least three levels are performed.

Other carriers

- 64479-Cervical transforaminal, single level

- 64481-51-Cervical transforaminal, each additional level

- 64475-51 - L/S facet joint nerve block, single level

- 64476-51 - L/S facet joint nerve block, each additional level

v.

Multiple lumbar transforaminal epidurals and multiple cervical facet joint nerve block

Physician

- $64483-$ L/S transforaminal epidural, single level

- $\quad 64484-$ L/S transforaminal, each additional level

- $\quad 64470-\mathrm{C} / \mathrm{T}$ facet joint nerve block, single level

- $64472-\mathrm{C} / \mathrm{T}$ facet joint nerve block, each additional level

- 76005 - Fluoroscopic guidance

Surgery center

Medicare

- Lumbar transforaminal epidurals and cervical facet joint nerve block codes are not approved procedures for surgery centers.

Other carriers

- $64483-\mathrm{L} / \mathrm{S}$ transforaminal epidural, single level

- 64484-51 - L/S transforaminal, each additional level

- 64470-51-C/T facet joint nerve block, single level

vi. Percutaneous lysis of adhesions and multiple cervical facet joint nerve blocks

Physician

- $62263-$ Percutaneous lysis of epidural adhesions

- $\quad 64470-\mathrm{C} / \mathrm{T}$ facet joint nerve block, single level

- 64472-51 (two units) - C/T facet joint nerve block, each additional level

- $\quad 76005$ - Fluoroscopic guidance 
Surgery center

Medicare

- Percutaneous lysis of adhesions and multiple cervical facet joint nerve block codes are not approved procedures for surgery centers.

Other carriers

- 62263 - Percutaneous lysis of epidural adhesions

- 64470-51 - C/T facet joint nerve block, single level

- 64472-51 (two units) - C/T facet joint nerve block, each additional level

vii. Bilateral lumbar facet joint nerve blocks and caudal epidural

Physician

Medicare

- 64475-50 - L/S facet joint nerve block, single level (bilateral)

- $\quad 64476$ - L/S facet joint nerve block, each additional level

- 62311-51 - L/S epidural

For carriers allowing bilateral billing

- 64475-50 - L/S facet joint nerve block, bilateral

- 64476-50 - L/S facet joint nerve block, each additional level

- 62311-51 - L/S epidural

For carriers not allowing bilateral billing

- 64475 - L/S facet joint nerve block, single level

- $\quad$ 64476 - L/S facet joint nerve block, each additional level

Surgery center

62311-51- L/S epidural

Medicare

- 64475-50 - L/S facet joint nerve block, single level (bilateral)

- 64476-51 - L/S facet joint nerve block, each additional level

- 62311-51 - L/S epidural

For carriers allowing bilateral billing

- 64475-50 - L/S facet joint nerve block, single level (bilateral)

- 64476-50 - L/S facet joint nerve block, each additional level

For carriers not allowing bilateral billing

62311- L/S epidural

- 64475-51 - L/S facet joint nerve block, single level
- 64476-51 - L/S facet joint nerve block, each additional level

\section{CONCLUSION}

Similar to death and taxes which are inevitable, ongoing changes in billing, coding and documentation, are also not only definite, but also unavoidable. Once again, it is accepted that CPT descriptive terms and identification of codes currently are the only means of documenting medical necessity and serve a wide variety of important functions in the field of medical practice including interventional pain management. Documentation of interventional pain procedures and subsequent billing and coding are of crucial importance not only in compliance with regulations, but also for good patient care, and finally, last but not least, for survival of the practice itself. This review has provided generally accepted practice patterns in a safe manner. However, caution must be exercised in utilizing the issues and guidelines mentioned in this article in your own practices.

\section{REFERENCES}

1. Manchikanti L. Appropriate documentation, billing and coding of interventional pain procedures. Pain Physician 2000; 3:218-236.

2. Manchikanti L. CPT 2000. Interventional pain management coding in the new millennium. Pain Physician 2000; 3:73-85.

3. Current Procedural Terminology, CPT 2000, Chicago, American Medical Association, 1999.

4. Medicare Program; revisions to payment policies under the physician fee schedule for Calendar year 2000. Final rule. HCFA 64 Federal Register, 59389, November 2, 1999.

5. Revisions to payment policies under the physician fee schedule for calendar year 2001, proposed rule HCFA Federal Register 65 (137), July 17, 2000.

6. Medicare Program; Prospective Payment System for Hospital Outpatient Services; Final Rule: Department of Health and Human Services. Health Care Financing Administration, 65 Federal Register 42 CFR Parts 412, 413, and 485, April 7, 2000.

7. Manchikanti L. State of interventional pain medicine. Pain Physician 2000; 3:241-255.

8. Program Memorandum - Department of Health and Human Services: Health Care Financing Administration, Transmittal No. AB - 00 - 28; May 2000.

9. Manchikanti L. Correct coding in interventional pain management. Pain Physician 2000; 3:313-321.

10. Manchikanti L. The impact of National Correct Coding Policy on interventional pain management. Pain Physician 1999; 2:33-45. 
11. National Correct Coding Primer 2000: Part 'B', News Group, Rockville, MD.

12. CCI edits require expertise in the face of voluminous outpatient, coding changes. HCPCS Report 2000; 14:15.

13. Manchikanti L. The role of evaluation and management services in pain management. Pain Physician 1999; 2:10-32.

14. Pain Physician News, Association of Pain Management Anesthesiologists, April 2000.

15. Anesthesia Answer Book Action Alert, UCG, Rockville, Maryland, May 2000; pp2-3.

16. Hines RL, Naulty, JS. Letter to Colleagues, Yale University School of Medicine, Department of Anesthesiology, May 16 ${ }^{\text {th }}, 2000$.
17. Medicare Program; Update of rate setting methodology, payment rates, payment policies, and the list of covered procedrues for Ambulatory Surgical Centers, proposed rule, HCFA, 63 Federal Register

18. HCFA's letter to Hon. Mitch McConnell from HCFA, July 21, 2000.

19. Manchikanti L, Singh V, Bakhit CE et al. Interventional techniques in the management of chronic pain. Part 1.0 Pain Physician 2000; 3:7-42.

20. Manchikanti L, Pampati V, Beyer C et al. The effect of neuraxial steroids on weight and bone mass density: A prospective evaluation. Pain Physician 2000; 3:357-366. 